the wide range of experimental and computational results, and many models contradict each other.

By means of large-scale atomistic simulations, Buehler and Gao show that hyperelasticity, the elasticity at large strains, can play a governing role in dynamical crack tip instabilities in the fracture of brittle materials. They report a modified instability model that treats the dynamical fracture instability as a competition between different mechanisms controlled by local energy flow and local stress field or atomic forces near the crack tip. Their results suggest that the fracture instability not only appears in materials with defects; it is an intrinsic phenomenon of dynamical fracture. See Figure 1 (p. 167).

"Our new theory reduces to existing models in limiting cases, but allows for a unified treatment of the instability problem applicable to a much wider range of materials," said Buehler. "We have discovered that the key to understanding the discrepancies in the literature is to consider the material behavior close to the breaking of bonds, rather than the material properties at small strains."
Most existing theories of fracture assume a linear elastic stress-strain law by only considering small strain deformation. However, the relation between stress and strain in real solids is strongly nonlinear due to large deformation near a moving crack tip, a phenomenon referred to as hyperelasticity or nonlinear elasticity.

The scientists have made another surprising discovery. "We find that elastic stiffening materials behavior, as found in rubber-like materials, can dramatically change the instability dynamics of cracks," Buehler said. Rubber is soft at small deformations, and becomes harder as the stretch is increased. "In such elastically stiffening materials, stable intersonic crack motion is possible," he said. These results are in contrast to existing theories, in which the speed of elastic waves is considered the limiting speed of fracture, analogous to the speed of light.

"We discovered that the classical theories of instability dynamics are only valid in a small range of material behavior," said Buehler. "In most real materials, the softening or stiffening close to bond breaking leads to a fundamental change in the instability dynamics, because energy flow is reduced or enhanced due to change in local wave speed."

These results represent a breakthrough in understanding how cracks propagate in brittle materials, and the findings could have wide impact in many scientific and engineering disciplines, said the researchers.

\section{Scanning Ultrasound Holography Allows Nanoscale Subsurface Imaging}

The characterization of deeply buried or embedded structures and features with lateral resolution of under $100 \mathrm{~nm}$ is critical in a number of science and engineering arenas. Subsurface features can be imaged with techniques such as acoustic microscopy, but with limited spatial resolution. More recently, scanning probe techniques, such as ultrasonic force microscopy, have been used for nanomechanical mapping of elastic and viscoelastic properties of surfaces. However, these techniques still lack the sensitivity required for imaging buried structures. Now, Gajendra Shekhawat

\title{
Announcement and Call for Papers
}

\section{October 17-19, 2006, in San Diego, California Advanced Metallization Conference (AMC) 2006}

This conference is the 23rd in a series devoted to leading-edge research in the field of advanced metallization and 3-D integration for IC applications. Papers may be submitted on topics affecting state-of-the-art and future directions in interconnect systems, including:

Metallization, material science, and interfaces Advanced deposition techniques and kinetics Nucleation and adhesion studies Diffusion barrier performance

Interconnect thin film microcharacterization Electrical and mechanical properties Morphology evolution and stability

Advanced semiconductor device architecture Low-k dielectrics

Metal and dielectric thin film barriers High-k and ferroelectric capacitors

Inductors, capacitors, etc., in the wiring levels

Advanced wiring schemes (e.g., for embedded DRAM, system-on-a-chip, etc.) Vertical (3-D) interconnects Nano-interconnects (CNT)

Metal gate transistors design, fabrication, characterization, and reliability

RF interconnects

Interaction of packaging with on-chip interconnects
Process modeling

Advancements in CVD and PVD deposition

Electrochemical and electroless deposition

Chemical mechanical polishing

Novel deposition and planarization techniques

Multilevel process integration issues

System-on-a-chip

3-D system integration

Novel interconnect system concepts

Chip interconnect/packaging interface issues

MEMS metallization issues and solutions

Advanced patterning and etching processes

Damascene and dual-damascene techniques

Barrier/liner/fill technology

Integration of $\mathrm{Cu}$ and $\mathrm{Al}$ with low-k dielectrics

Reliability and performance data and simulations

Dual damascene process defects

Electromigration and stress migration

Dielectric reliability and diffusion studies

Adhesion, corrosion, and other stress testing

Reliability of active chips with Cu and/or low-k

Bonding and packaging issues for advanced interconnects
Abstracts are due June 2, 2006.

Send abstracts (two pages, 500 words, with supporting figures on second page) to Jenny Black Deer, UC Berkeley Extension, 1995 University Ave., Berkeley, CA 94720-7010; fax: (510) 642-6027; e-mail: amc@unex.berkeley.edu. Please note: all abstracts submitted electronically must be a pdf file (all fonts must be embedded in the file). Include the author's name, affiliation, mailing address, e-mail address, and phone and fax numbers on the abstract.

For an announcement brochure:

Call (510) 642-4151, fax (510) 642-6027, e-mail amc@unex.berkeley.edu, or write to: Continuing Education in Engineering, UC Berkeley Extension, 1995 University Ave., Berkeley, CA 94720-7010. Or visit www.unex.berkeley.edu/eng/metal
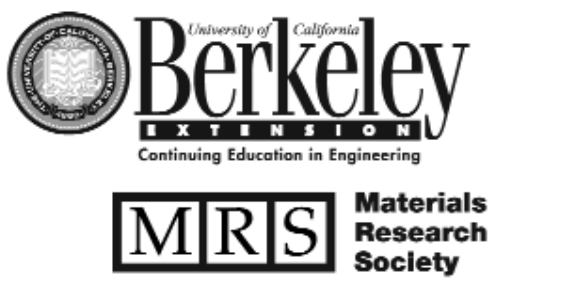

An MRs-affiliated meeting

For more information, see http://Www.mrs.org/bulletin_ads 
and Vinayak Dravid from Northwestern University have reported the development of scanning near-field ultrasound holography (SNFUH) that allows characterization of depth information as well as spatial resolution in the $10-100-\mathrm{nm}$ range. The method can be used to image buried nanostructures in diverse materials systems including microelectronic structures and biological samples. They report the technique and results in the October 7 , 2005, issue of Science (p. 89; DOI: $10.1126 /$ science.1117694).

SNFUH is based on scanning probe microscopy (SPM). A high-frequency acoustic wave, on the order of a megahertz or more, is applied to the bottom of a specimen, while a second wave is launched on the SPM cantilever at a slightly different frequency. The interference of these two waves forms a surface acoustic standing wave. Buried features perturb the specimen acoustic wave, and these perturbations to the phase and amplitude of the surface acoustic standing wave are monitored by the SPM cantilever. A pictorial representation of the acoustic wave perturbation is recorded
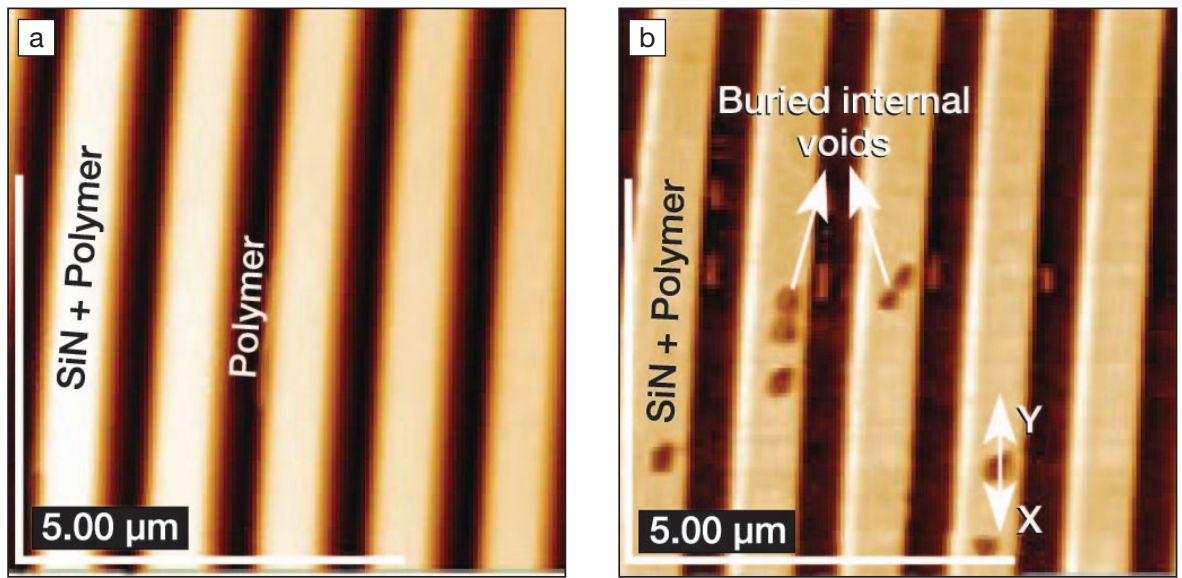

Figure 1. (a) Typical atomic force microscope topography image shows a low-dielectric polymer coating covering trenches in a model microelectronics trench structure. (b) The corresponding scanning near-field ultrasound holography phase image reveals embedded voiding in the polymer coating over the nitride. Reprinted with permission from Science (Oct. 7, 2005) p. 89. (C2005 AAAS.

that includes quantitative information about the internal features. The researchers used a model polymer-gold nanoparticle composite to demonstrate the spatial resolution and depth sensitivity of the technique. The nanoparticles with an average diameter of $15 \mathrm{~nm}$ were buried beneath a 500-nm-thick polymer

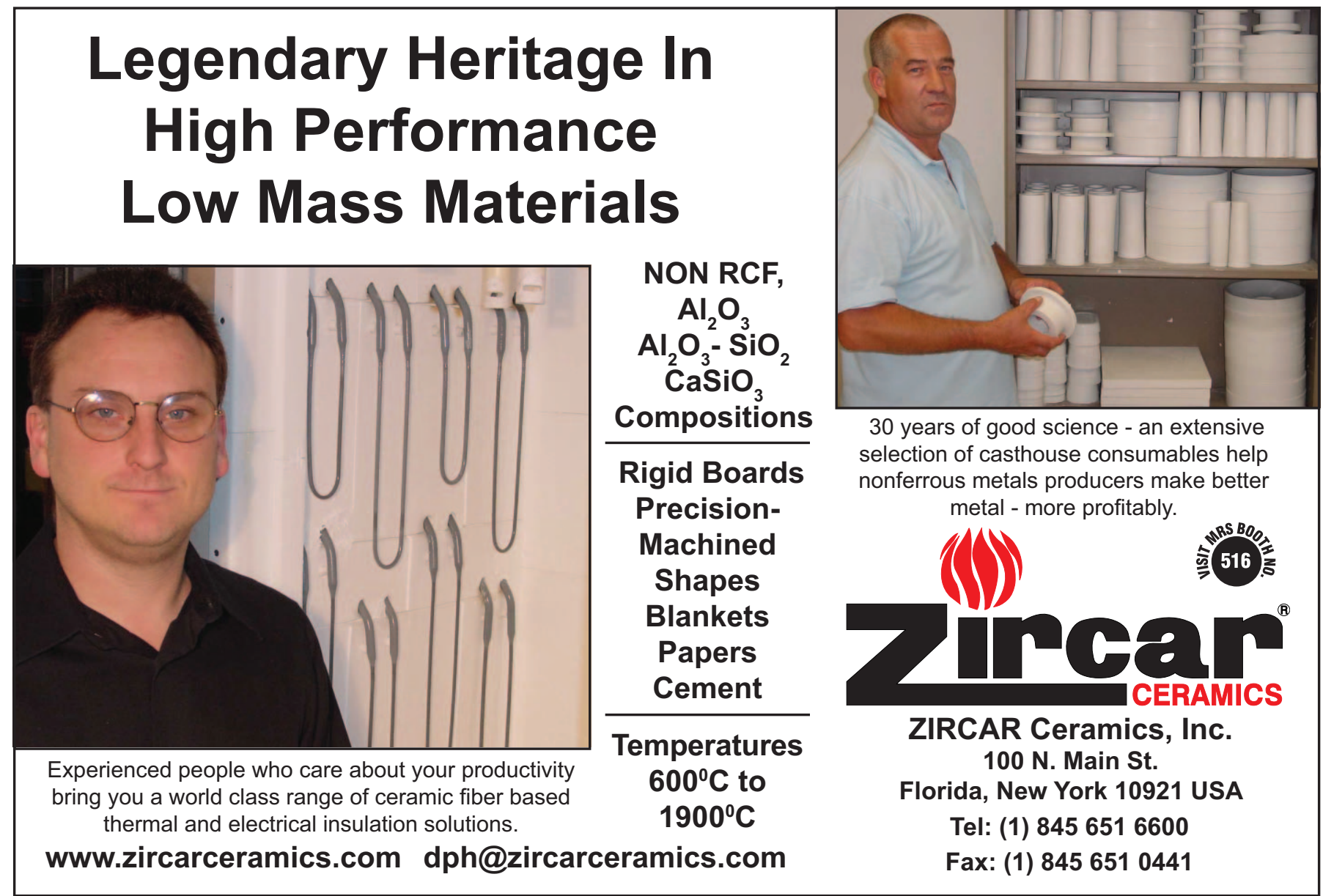

For more information, see http://www.mrs.org/bulletin_ads 
cover layer on a silicon substrate. While normal atomic force microscopy (AFM) showed a smooth, featureless surface of the top polymeric layer, the SNFUH phase image clearly showed the dispersed, buried gold nanoparticles.

SNFUH is particularly well suited for detecting defects and voids in microelectronics structures. For example, the scale of fabrication continues to shrink and interconnect metal lines are approaching 60-nm widths. Model shallow trench structures were fabricated in a dielectric material with a 50-nm-thick silicon nitride layer deposited as a capping layer and etched into the trenches. A 500-nm-thick lowdielectric polymer layer was deposited by spin-coating and annealing. A conventional topography scan showed a uniform and continuous polymer coating at the bottoms of the trenches as well as on the tops of the lines, as seen in Figure 1 (p. 169). However, the corresponding SNFUH phase image revealed embedded voids within the polymer and at the $\mathrm{SiN}$-polymer interfaces.

The SNFUH technique can also be used to image embedded or buried sub- structures in biological samples. A nearcontact mode was used for imaging soft structures, wherein the probe tip is lifted by $2-5 \mathrm{~nm}$ after it touches the surface. This was used to obtain high-contrast, high-resolution images of malaria parasites inside infected red blood cells. The direct real-space in vitro imaging was performed without labeling or sectioning the cells under physiologically viable conditions. SNFUH was thus demonstrated to be a versatile technique for nondestructive, high-resolution, real-space imaging of diverse materials systems. In particular, it fills the spatial resolution gap at the 10-100-nm scale for nondestructive subsurface imaging.

GOPAL RAO

\section{Silica Nanoparticles Doped with Multiple Fluorescent Dyes Demonstrated as Potential New Barcoding Tags}

Often in biological imaging experiments, it is desirable to view several components of a structure simultaneously in real time. This can be done with small-molecule fluo- rescent tags, but this technique is limited by the number of available dyes that are excited at the same wavelength but have distinguishable emission spectra. Quantum dots, polymer microspheres, and other materials have been used to overcome this limitation and create "bio-barcoding" systems, but many of these have problems with performance. In the January 11 issue of Nano Letters (p. 84; DOI: 10.1021/ n1052105b), researchers L. Wang and $W$. Tan from the University of Florida at Gainesville have presented a novel solution to this problem. The researchers incorporated combinations of three common fluorophores into silica nanoparticles (NPs) to create NPs that respond to monochromatic illumination with a range of distinguishable emission signatures. They also demonstrated the potential for using these materials as tags for biological molecules by functionalizing the NPs with biotin and binding them to avidinfunctionalized microspheres.

The researchers incorporated the organic dyes FITC, R6G, and ROX into silica NPs in a variety of ratios. Alone, these dyes

\section{www.mrs.org}

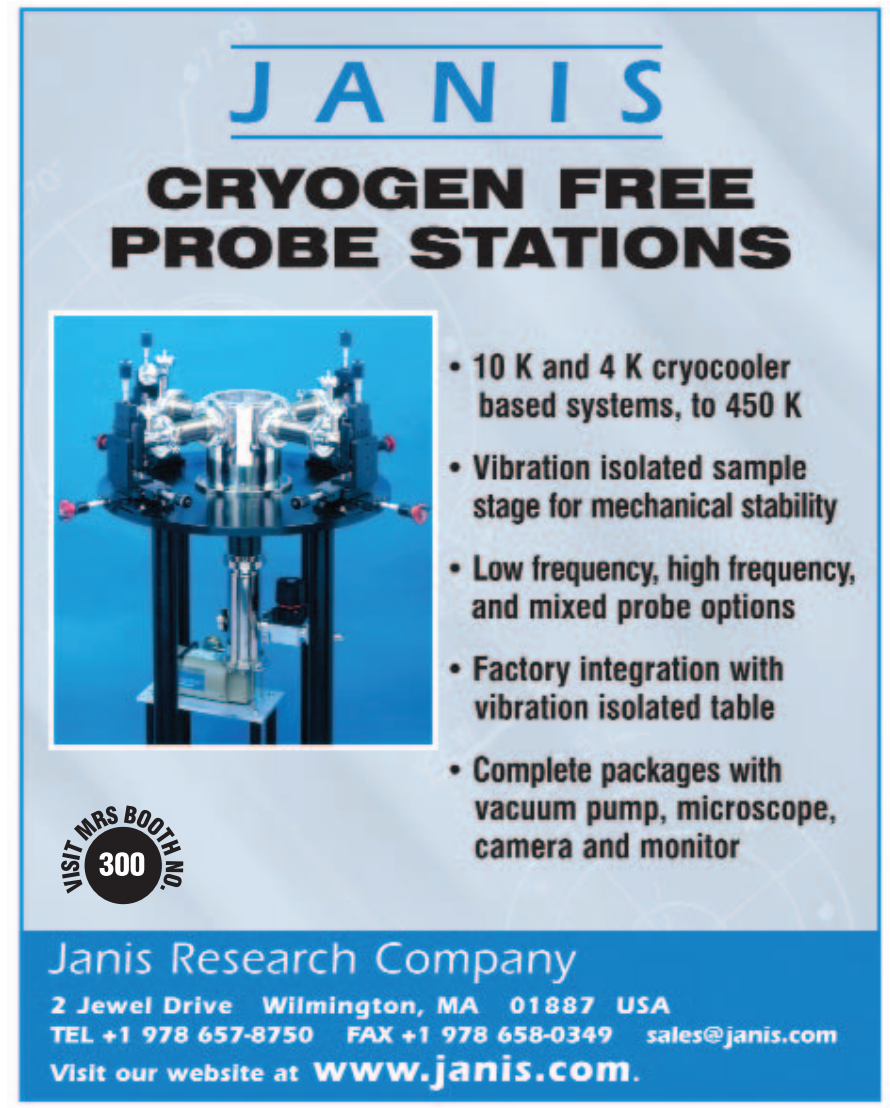

For more information, see http://www.mrs.org/bulletin_ads

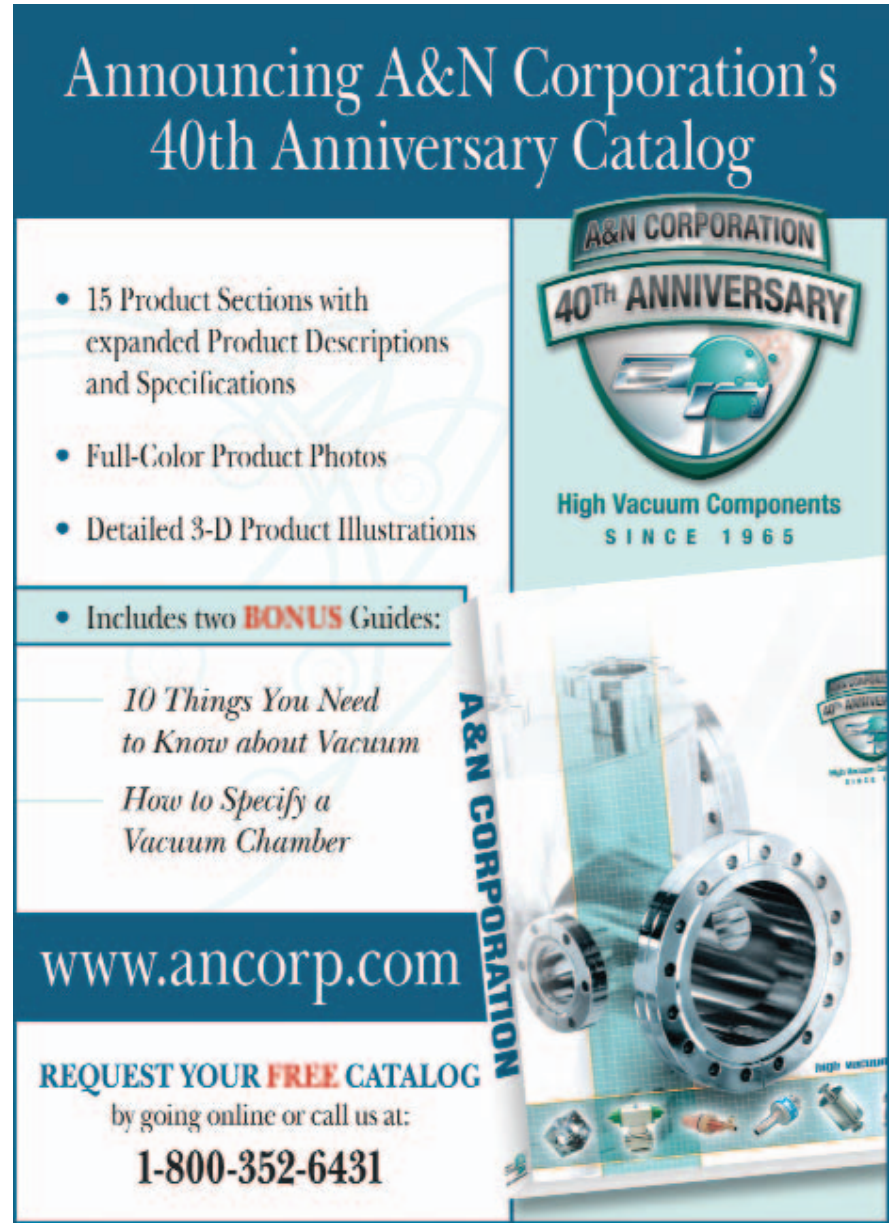

For more information, see http://www.mrs.org/bulletin_ads 